\title{
The Energetics of a Collapsing Meridional Overturning Circulation
}

\author{
ANDREW MCC. HOGG \\ Research School of Earth Sciences, and ARC Centre of Excellence for Climate System Science, \\ The Australian National University, Canberra, Australian Capital Territory, Australia \\ HenK A. DiJKSTRA \\ Institute for Marine and Atmospheric Research, Department of Physics and Astronomy, \\ Utrecht University, Utrecht, Netherlands \\ JUAN A. SAENZ \\ Research School of Earth Sciences, The Australian National University, Canberra, \\ Australian Capital Territory, Australia
}

(Manuscript received 29 October 2012, in final form 25 March 2012)

\begin{abstract}
A well-studied example of natural climate variability is the impact of large freshwater input to the polar oceans, simulating glacial melt release or an amplification of the hydrological cycle. Such forcing can reduce, or entirely eliminate, the formation of deep water in the polar latitudes and thereby weaken the Atlantic meridional overturning circulation (MOC). This study uses a series of idealized, eddy-permitting numerical simulations to analyze the energetic constraints on the Atlantic Ocean's response to anomalous freshwater forcing. In this model, the changes in MOC are not correlated with the global input of mechanical energy: both kinetic energy and available potential energy (APE) increase with northern freshwater forcing, while the MOC decreases. However, a regional analysis of APE density supports the notion that local maxima in APE density control the response of the MOC to freshwater forcing perturbations. A coupling between APE input and changes in local density anomalies accounts for the difference in time scales between the recovery and collapse of the MOC.
\end{abstract}

\section{Introduction}

The processes that drive ocean circulation, and particularly the ocean's meridional overturning circulation (MOC), remain a topic of debate. A key aspect of this debate is the source of the global ocean mechanical energy budget and can be illustrated using the "horizontal convection" paradigm for the ocean overturning circulation (Hughes and Griffiths 2008). In this paradigm, one delineates the energetic contributions from surface buoyancy forcing (which generates regions of dense and less dense fluid) from the flow induced by

Corresponding author address: Andrew Hogg, Research School of Earth Sciences, The Australian National University, Canberra, ACT 0200, Australia.

E-mail: Andy.Hogg@anu.edu.au mechanical stresses on the boundary (predominantly wind stresses and tidal motion).

It is now widely recognized that, as argued by Munk and Wunsch (1998), the ocean's stratification is only equilibrated if the advective transport of density into the ocean interior (i.e., the sinking of dense water into the abyssal ocean) is balanced by downward turbulent diffusion of buoyancy. In the limit approaching zero vertical diffusion it is argued that the dissipation of this flow tends to zero (Paparella and Young 2002), implying that buoyancy forcing at a single level (i.e., the ocean surface) contributes a negligible amount of mechanical energy into the circulation (Wunsch and Ferrari 2004). Thus, it has been inferred that the primary source of energy for the ocean's circulation is mechanical stresses (winds and tides) on the ocean boundaries (Kuhlbrodt et al. 2007).

A contrary view to the "mechanically driven" argument has emerged in a series of recent studies (e.g., 
Hughes et al. 2009; Tailleux 2009). If one defines the mechanical energy to be the superposition of kinetic energy (KE) and available potential energy (APE), then it can be demonstrated that buoyancy forcing, even when applied at a single surface, generates mechanical energy through APE (Hughes et al. 2009). Numerical ocean models (with finite vertical diffusion) indicate that the energy from surface buoyancy forcing is likely to be the same order of magnitude as wind stress (Saenz et al. 2012). Moreover, as described in Saenz et al. (2012), there is a feedback between the power inputs from each energy source that is facilitated by the Antarctic Circumpolar Current (ACC). The primary site of wind power input occurs where ocean surface velocities are aligned with wind stress, meaning that a disproportionate amount of energy input is derived from the Southern Ocean (e.g., Hughes and Wilson 2008). Energy input from surface buoyancy forcing is maximized by increasing the density of the densest waters, which is also most likely to occur in the Southern Ocean (Saenz et al. 2012). Strengthening winds in the Southern Ocean directly increase wind power input, but also enlarge the area of dense water exposed to strong buoyancy loss, thereby enhancing surface buoyancy forcing power input. Likewise, stronger buoyancy forcing directly increases power input from surface buoyancy forcing, but also increases ACC transport (e.g., Borowski et al. 2002; Hogg 2010) thereby enhancing wind-current correlations and resulting in greater wind power input.

Thus, there is evidence supporting the notion that, in today's ocean at least, surface buoyancy forcing contributes a substantial fraction of the energy of the circulation. The extent to which this result applies in the limit of small vertical diffusion is currently unclear, but there are some arguments that the results of Paparella and Young (2002) may be overly restrictive (Scotti and White 2011), and that dissipation is not dynamically linked with the strength of the MOC (Gayen et al. 2013).

The extent to which mixing controls the overturning circulation also depends upon the interhemispheric nature of the overturning cell. By progressively lowering vertical diffusivity in numerical simulations of ocean circulation and extrapolating to the limit of zero diffusivity, Toggweiler and Samuels (1998) suggest that an upper overturning cell associated with the formation of North Atlantic Deep Water (NADW) could exist, owing to wind-driven upwelling in the latitudes of the Southern Ocean. Thus, according to this extrapolation, it could be possible to have an adiabatic pole-to-pole circulation (Wolfe and Cessi 2011) with minimal energy input by the wind only, provided that the northern and southern parts of the basin share an outcropping isopycnal.
However, even in this hypothetical limit, buoyancy does net work in the sinking branch of the NADW cell.

Of course, the purpose of these, at times, arcane arguments is to foster a better understanding of how the ocean circulation is likely to respond to future changes in forcing. An example of how surface forcing may modulate the global energy balance of the oceanic circulation is the weakening or shutdown of the NADW cell of the overturning circulation caused by freshwater fluxes from (for example) glacial meltwater release. This problem derives in part from paleoceanographic inferences of overturning circulation variability on millennial time scales (Clark et al. 2002) and is also important in projections of future climate states. Standard test cases to understand this problem using ocean models are the so-called freshwater hosing experiments (e.g., Rahmstorf 1995; Stouffer et al. 2006). In these experiments, an amount of freshwater is introduced into the northern Atlantic Ocean, ostensibly representing the release of glacial meltwater or other changes in the hydrological cycle. The freshwater perturbation leads to a reduction of NADW formation resulting, in most cases, in a cessation of the MOC. Northward heat transport decreases, leading to large reductions in surface air temperature in the Northern Hemisphere (Stouffer et al. 2006; Smith and Gregory 2009).

Under the right conditions, one can recover elements of the prediction of Stommel (1961) that alternative equilibrium states, including one with no NADW formation, can occur. For example, Stouffer et al. (2006) use a range of models to demonstrate that small temporary freshwater perturbations $[\sim 0.1 \mathrm{~Sv}(1 \mathrm{~Sv} \equiv$ $\left.\left.10^{6} \mathrm{~m}^{3} \mathrm{~s}^{-1}\right)\right]$ lead to quick recovery, while large perturbations $(\sim 1 \mathrm{~Sv})$ generate sudden cessation of the MOC with a more gradual return once the perturbation is removed. Hawkins et al. (2011) generate bistability in the fully coupled FAMOUS model and support the indicator of Huisman et al. (2010) for the condition of bistability-based on the sign of freshwater transport by the MOC at the southern boundary of the Atlantic Ocean, although it's not clear that this indicator will extend to eddy-permitting models (den Toom et al. 2013, manuscript submitted to J. Phys. Oceanogr.).

Thus, we contend that the arguments surrounding the energy budget of the equilibrium ocean can be further illuminated using transient cases such as the freshwater hosing experiment. In particular, the widely acknowledged variation of the MOC in response to perturbations in surface buoyancy forcing implies that the buoyancy forcing plays both a dynamical and an energetic role in governing the circulation. In this paper we take an idealized, eddy-permitting model of the pole-topole ocean overturning circulation (see section 2), and 
(a) Bathymetry

(b) Wind

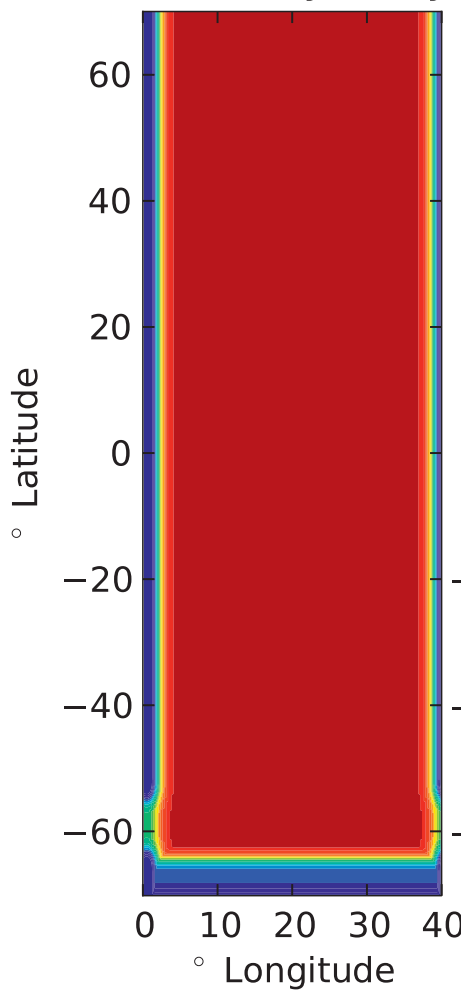

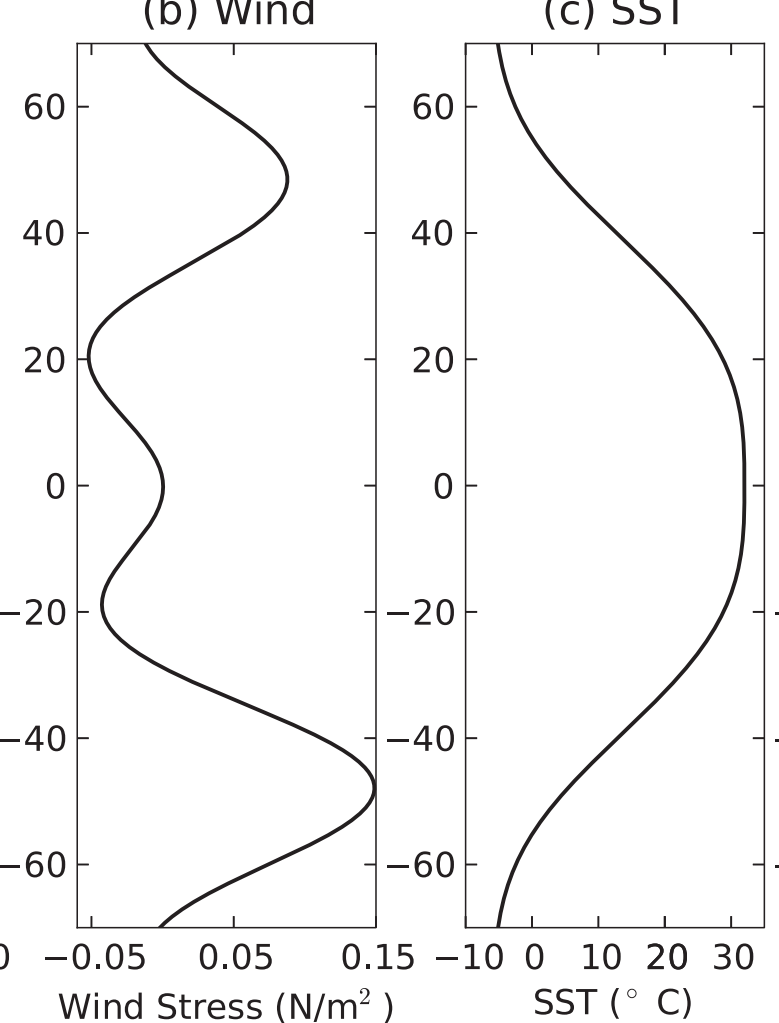

(d) Freshwater

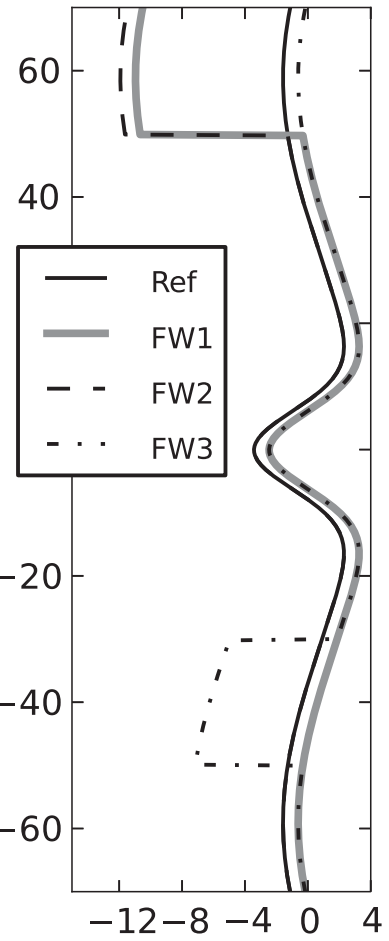

FW Flux $\left(\times 10^{-8} \mathrm{~m} / \mathrm{s}\right)$

FIG. 1. (a) Model bathymetry (range 0-4000 m, contour interval $500 \mathrm{~m}$ ), (b) zonal wind stress forcing as a function of latitude, (c) sea surface temperature relaxation field, and (d) freshwater flux (evaporation minus precipitation) for four different experiments.

apply perturbations to the surface buoyancy forcing in the form of imposed freshwater flux anomalies. We investigate the dynamical response to several different perturbations (section 3); however, we will concentrate on reconciling these dynamics with an analysis of global and regional energy budgets using the APE framework (section 4). Our goal is to quantify the key energy sources associated with the response of the MOC to changes in surface buoyancy forcing.

\section{Model}

We conduct a series of simulations in a sector configuration mimicking the Atlantic Ocean, using the Massachusetts Institute of Technology General Circulation Model (MITgcm) in hydrostatic, Boussinesq mode (Marshall et al. 1997). The domain is $40^{\circ}$ in longitudinal extent and covers the latitudes $70^{\circ} \mathrm{S}-70^{\circ} \mathrm{N}$. With $1 / 4^{\circ}$ resolution and square grid boxes (i.e., Mercator projection) at all latitudes, the model can be considered as eddy permitting, and no explicit eddy parameterization scheme is used. Horizontal and vertical viscosity/diffusion are minimized (with a biharmonic lateral viscosity/diffusivity of $10^{11} \mathrm{~m}^{4} \mathrm{~s}^{-1}$ and a vertical viscosity/diffusivity of $10^{-5} \mathrm{~m}^{2} \mathrm{~s}^{-1}$ ). The vertical diffusivity is amplified by a factor of 200 near the surface to generate mixed layers of an appropriate depth. The model uses a linear equation of state where the density depends upon both temperature and salinity.

The model bathymetry is a simple box of maximum depth $4000 \mathrm{~m}$ with sloping sidewalls and a sloping boundary in the south, as shown in Fig. 1a. The single point of departure from rectangular geometry is a circumpolar channel centered on $60^{\circ} \mathrm{S}$ with a simple sill of depth $1800 \mathrm{~m}$, designed to represent the Southern Ocean and the Drake Passage. The reference case is forced by constant, prescribed wind stress (Fig. 1b), relaxation of sea surface temperature to a constant, latitudinally symmetric profile (Fig. 1c; restoring time scale of 60 days), and prescribed freshwater fluxes (solid line in Fig. 1d), derived from the freshwater forcing used in Huisman et al. (2012).

The reference case is run from initial conditions for 1680 years, by which stage key flow metrics (circumpolar transport, overturning streamfunctions, and bottom density) and energy reservoirs (total kinetic and available potential energy) are very close to equilibrium. At this time (designated year 0 in the subsequent analysis), we branch off three cases with perturbed freshwater 

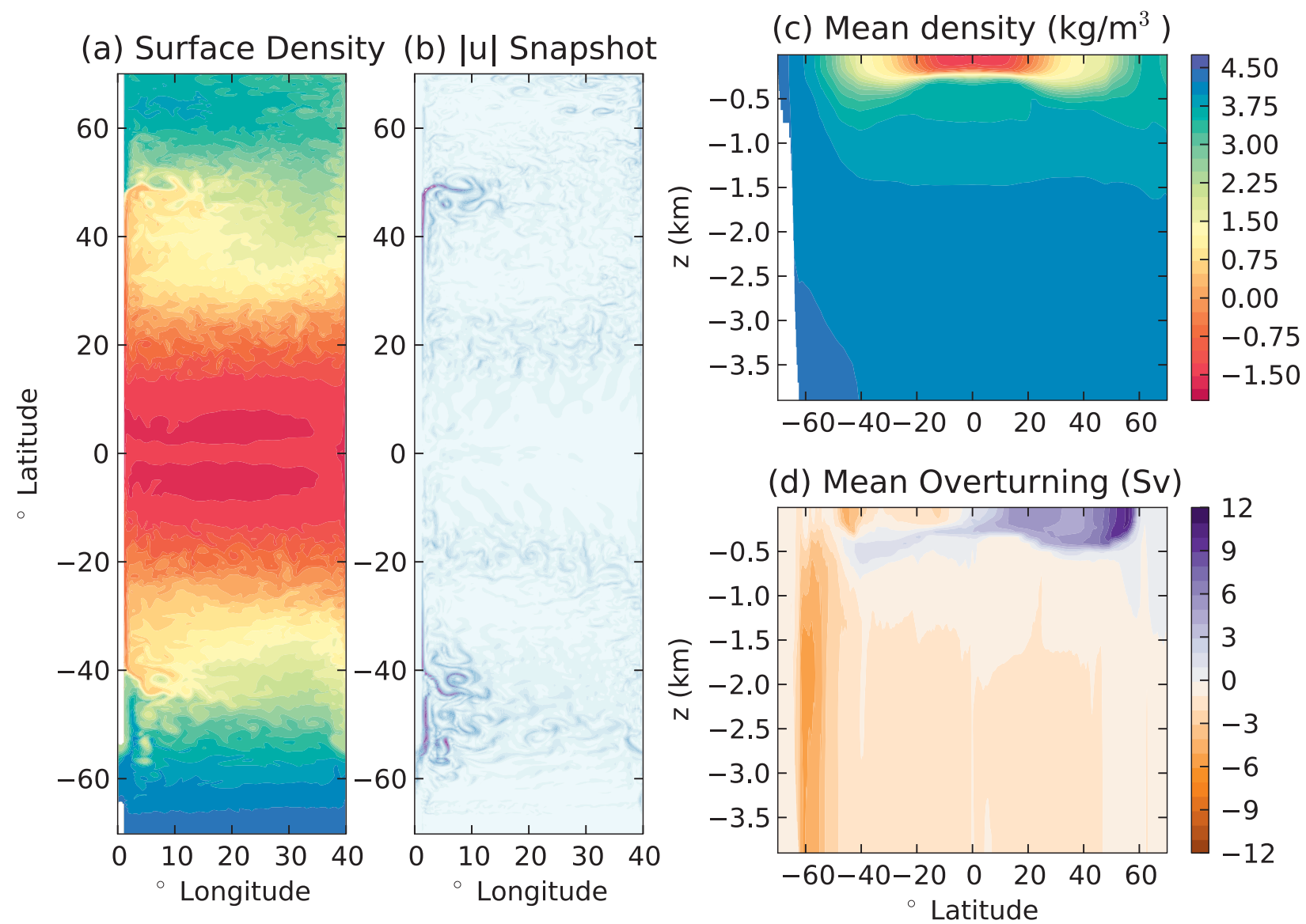

FIG. 2. Equilibrium state of the reference case. (a) Snapshot of sea surface density [plotted as the anomaly from the reference density and using the same color scale as (c)], (b) snapshot of the absolute magnitude of the horizontal velocity (color scale ranging from 0 to $2.4 \mathrm{~m} \mathrm{~s}^{-1}$ ), (c) zonal- and time-averaged density field as a function of height $z$, and (d) zonal- and time-averaged overturning circulation evaluated on density surfaces and remapped into physical space. Averages are taken over the last 100 years of the perturbation (i.e., years 300-400 in Fig. 3).

profiles (shown in Fig. 1d). Case FW1 (heavy gray line) is a standard "freshwater flooding" perturbation equivalent to $0.5 \mathrm{~Sv}$ of freshwater flux (relative to the reference case) into the northern part of the domain but with compensating fluxes at other latitudes so that the surface integrated net freshwater forcing is zero. This salinity compensation case represents the forcing that would be experienced if freshwater flux anomalies were generated by changes in the hydrological cycle, where there is no net freshwater input to the ocean. Case FW2 (dashed line) uses a $0.5 \mathrm{~Sv}$ freshwater perturbation, but with no compensation at other latitudes, meaning that there is a monotonic freshening of the model ocean. This case represents the scenario expected from a glacial meltwater input to the northern Atlantic Ocean. The freshening trend is expected to generate a different partitioning of energy between background and available potential energy from the FW1 case where global-average salinity is constant. The third case, FW3 (dash-dot line in Fig. 1d), uses the same size perturbation as FW1 but the perturbation is located in the southern part of the domain. Salinity compensation is used, as with FW1; this case therefore represents an amplification of the hydrological cycle that results in greater freshwater input at the latitudes of the Antarctic Circumpolar Current.

Each perturbed case FW1-FW3 is run for 400 years, during which time the behavior of the MOC is monitored. Subsequently, we return to the reference freshwater forcing and integrate for another 400 years to determine the recovery of the MOC after the earlier imposed perturbations.

\section{Dynamical response to freshwater perturbations}

The state of the reference case at equilibrium is shown in Fig. 2. Snapshots of surface density (relative to the 
reference density $\rho_{0}$; Fig. 2a) and the magnitude of the horizontal velocity (Fig. 2b) show the general character of the surface circulation. Strong western boundary currents in both hemispheres give rise to substantial eastward jets and eddy generation in the midlatitudes. In addition, this model supports a strongly eddying circumpolar current that shields the polar southern latitudes from ocean heat/freshwater transport; as a result, despite latitudinally symmetric SST relaxation and freshwater fluxes, density in the southern region is substantially greater than in the north (Fig. 2a). Abyssal density is thus positively correlated with Southern Ocean mixed layer density (Fig. 2c), where the thickness of the mixed layer is strongly controlled by the vertical profile of the specified vertical diffusion.

The residual overturning streamfunction, that is, the MOC calculated on density surfaces and interpolated back into height coordinates (Wolfe and Cessi 2011), averaged over years 300-400 of the simulation is shown in Fig. 2d. Here, we see a dominant abyssal cell fed by southern dense water formation. The overturning in the Northern Hemisphere is large in magnitude, but shallow in extent [a typical problem in these sector types of models—see Jones et al. (2011), Munday et al. (2013), and Shakespeare and Hogg (2012)]. A small fraction of this northern overturning crosses the equator to upwell in the Southern Ocean; this component of the overturning is consistent with the notion of an adiabatic poleto-pole circulation (Wolfe and Cessi 2011).

Figure 3 shows time series of the magnitude of the primary current systems as a function of time for each simulation. Here, the circumpolar transport $T_{\mathrm{ACC}}$ is the total eastward volume transport through the circumpolar channel; the northern overturning $\psi_{N}$ is the maximum value of the residual overturning streamfunction north of $40^{\circ} \mathrm{N}$; southern upwelling $\psi_{\mathrm{SO}}$ is the minimum value of the residual overturning streamfunction averaged between $20^{\circ}$ and $30^{\circ} \mathrm{S}$; and the abyssal overturning $\psi_{S}$ is the maximum of the residual overturning streamfunction south of $20^{\circ} \mathrm{S}$ with density exceeding $3.0 \mathrm{~kg} \mathrm{~m}^{-3}$ (relative to $\rho_{0}$ ). Note that from year 400 , the freshwater forcing returns to that of the reference state. The structure of both the stratification and residual overturning circulation for each of the perturbed cases (averaged over years 300-400) is shown in Fig. 4.

Freshwater forcing anomalies in the northern part of the basin with salinity compensation (FW1; heavy gray line in Fig. 3) result in a near-immediate shutdown of the northern overturning (Fig. 3b). The residual overturning streamfunction drops from $\sim 12$ to $\sim 4 \mathrm{~Sv}$ and is roughly constant throughout the perturbation. Moreover, the overturning cell is almost eliminated from the surface layers (Figs. 4a,b), implying that the $4 \mathrm{~Sv}$ of overturning may be a memory effect of the earlier forcing. Upwelling in the Southern Ocean also decreases (Fig. 3c) - it should be noted that shared isopycnal conditions for adiabatic pole-to-pole overturning (Wolfe and Cessi 2011) are no longer satisfied. Circumpolar transport (Fig. 3a) shows a moderate increase, while the abyssal southern overturning remains fairly steady for the first 300 years after which a sudden pulse occurs in the years 300-400 (Fig. 3d).

A freshwater anomaly without compensation (FW2; dashed line) has a broadly similar effect on the northern overturning and abyssal circulation. However, the southern upwelling is more persistent, and circumpolar transport follows a near-linear trend throughout the perturbation rising to almost double the reference value after 400 years. In short, this effect appears to be influenced by a steady buildup of the north-south salinity gradient throughout the perturbation, which is only weakly mixed across the circumpolar current and drives a stronger circumpolar flow via the thermal wind relation.

Applying the perturbation (with salinity compensation) in the Southern Ocean (FW3; dash-dot line) results in enhancement of the upper cell in both hemispheres (Fig. 4f) because of the role of surface buoyancy forcing in controlling the net upwelling in the Southern Ocean [as predicted by Morrison et al. (2011), and as shown in Fig. 3c]. The perturbation acts to increase the abyssal overturning, while the circumpolar transport is relatively unaffected.

In the FW1 and FW3 cases, the perturbed overturning circulation returns to the original state within 300 years once the forcing returns to the reference conditions. In the FW2 case, the salt content has changed leading to a different state than the reference state. For all three cases, the model does not reach a new equilibrium with a weak MOC circulation as could possibly exist (Stommel 1961), despite the large forcing perturbations applied. However, there is a significant asymmetry in the rate of change of the overturning consistent with many of the models in the intercomparison of Stouffer et al. (2006); reductions in the upper overturning cell occur within decades but take centuries to reestablish. The circumpolar current appears to be governed by very different mechanisms, with a more persistent transport anomaly, which shows no signs of relaxing after the perturbed forcing is removed.

The dynamical response of this model is broadly consistent with previous simulations in eddying global ocean models (Marsh et al. 2010; Weijer et al. 2012), although the integration time is much longer here. The northern overturning cell is strongly dependent on local forcing, and if this overturning cell is shut down it directly influences the wind-driven upwelling in the 


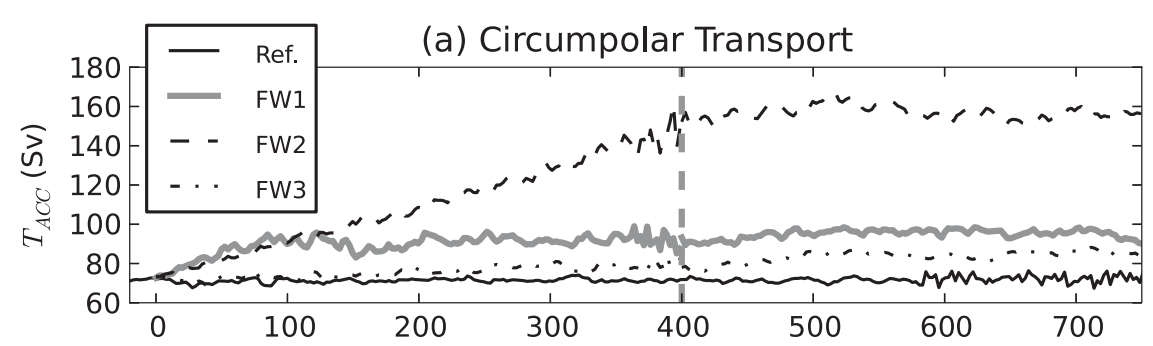

(b) Northern Overturning Streamfunction

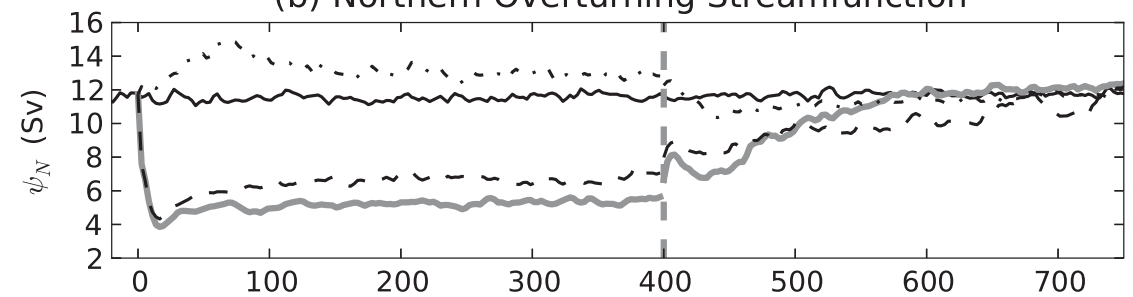

(c) Southern Upwelling

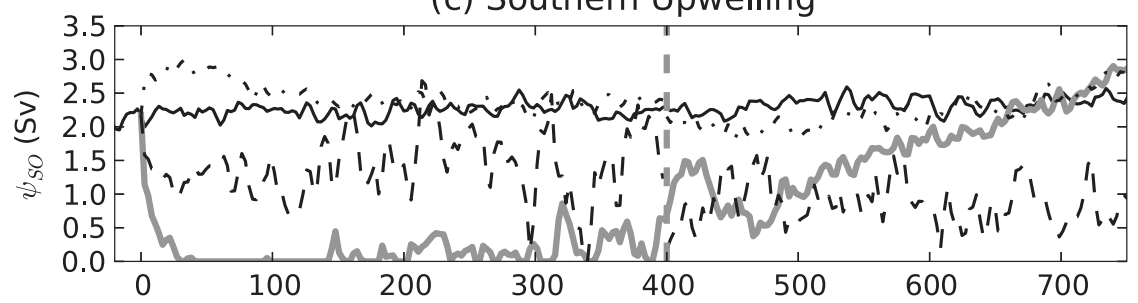

(d) Abyssal Southern Overturning

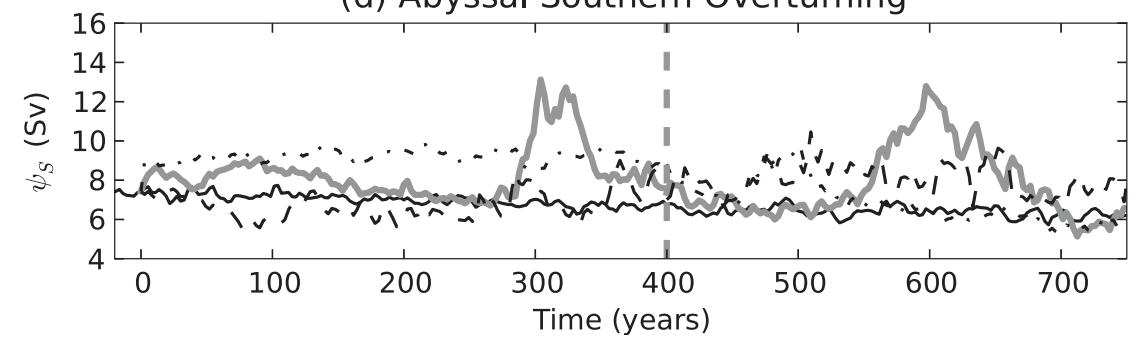

FIG. 3. Time series of transports in the four simulations. (a) Circumpolar transport, (b) Northern Hemisphere overturning streamfunction, (c) rate of upwelling in the circumpolar current latitudes, and (d) rate of formation of abyssal water in the southern part of the domain. Perturbations to the freshwater forcing are switched on at year 0 (after 1680 years spinup) and revert to the original forcing at year 400 , indicated by the vertical dashed gray line.

Southern Hemisphere. Conversely, freshwater flux in the Southern Ocean amplifies the upwelling in that region and enhances the northern overturning cell. The abyssal southern overturning circulation is not strongly influenced by the perturbations, although higher variability is present in some cases, and the circumpolar current increases in all cases, due largely to amplification in the meridional salinity gradient. While there is an asymmetry in the response of the upper overturning cell, none of these cases shows indications for the existence of multiple equilibria.

\section{Energetics}

The simulations outlined above produce significant responses in the ocean overturning circulation, providing test cases to examine the nature of the power sources driving changes in the overturning circulation. To achieve this, we first examine the global energy balance, and then proceed to look at regional variations in power sources.

\section{a. Global energy balance}

Following Hughes et al. (2009), we define the global mechanical energy budget to be the combination of kinetic energy and APE. Kinetic energy (per unit mass) $E_{k}$ is defined to be

$$
E_{k}=\frac{1}{2 V} \int_{V} u_{i} u_{i} d V
$$


(a) FW1 Density

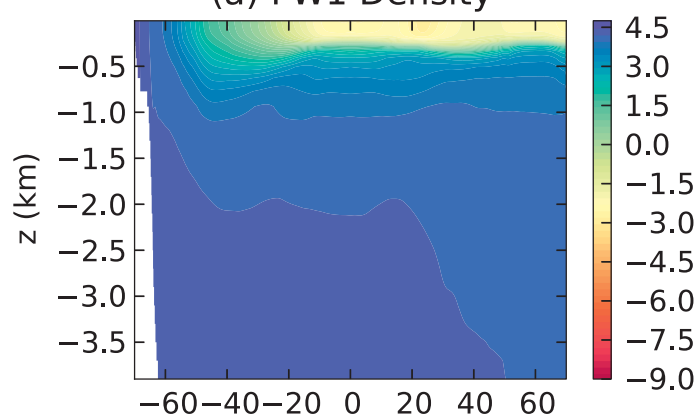

(c) FW2 Density

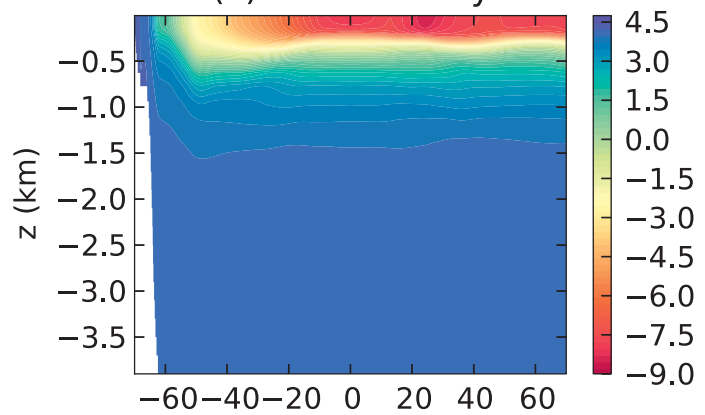

(e) FW3 Density

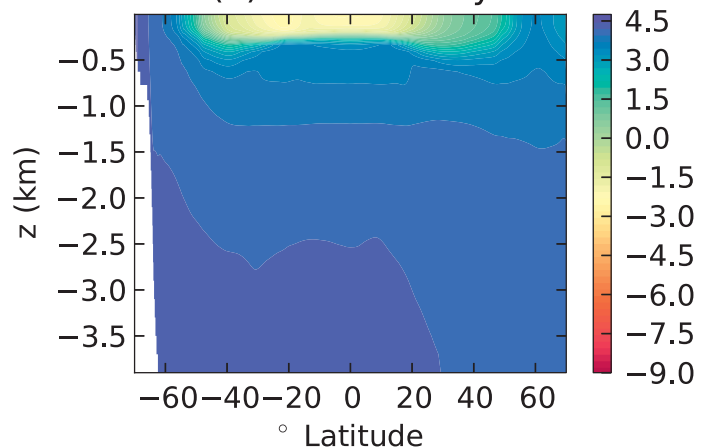

(b) FW1 Overturning

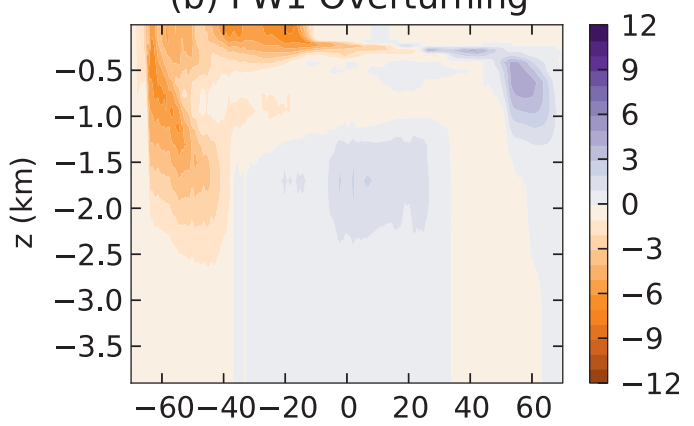

(d) FW2 Overturning

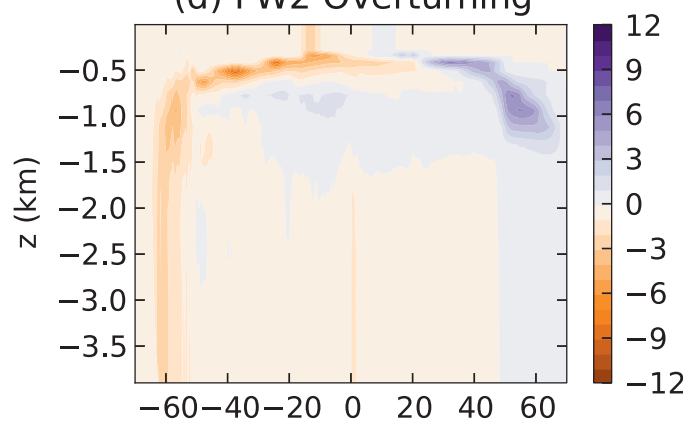

(f) FW3 Overturning

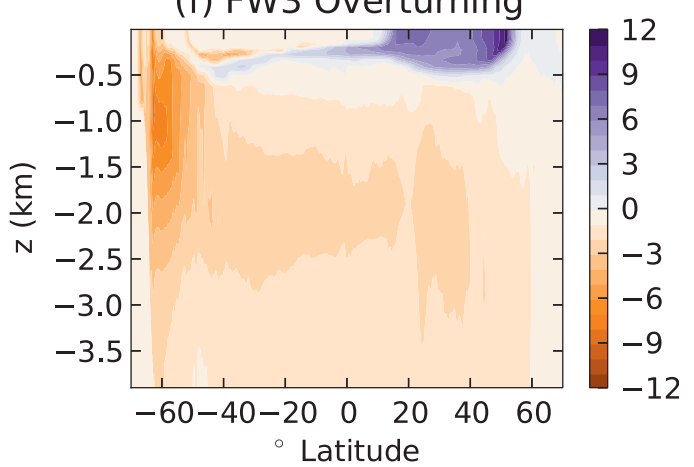

FIG. 4. (a),(c),(e) Zonal- and time-averaged density $\left(\mathrm{kg} \mathrm{m}^{-3}\right.$; note different color scale to Fig. 2c) and (b),(d),(f) overturning streamfunction (Sv) for the three simulations with freshwater forcing perturbations: FW1 in (a),(b); FW2 in (c),(d); and FW3 in (e),(f). Averages are taken over the last 100 years of the perturbation (i.e., years 300-400 in Fig. 3).

where $V$ is the volume of the ocean, $u_{i}$ is the velocity in component direction $i$ and repeated indices imply summation. We write the kinetic energy tendency of the ocean model as

$$
\frac{d E_{k}}{d t}=\Phi_{\tau}-\Phi_{z}-\epsilon
$$

where

$$
\Phi_{\tau}=\frac{1}{V} \int_{S} u_{i} \tau_{i} d S
$$

is the power input from the wind stress $\left(\tau_{1}=\tau^{x}, \tau_{2}=\tau^{y}\right)$ and depends upon the correlation between wind stress and ocean surface velocity;

$$
\Phi_{z} \equiv \frac{g}{\rho_{0} V} \int_{V} \rho w d V
$$

is the conversion of energy between potential and kinetic energy; and

$$
\epsilon \equiv \frac{1}{V} \int_{V} K_{\nu}\left(\frac{\partial u_{i}}{\partial x_{j}}\right)^{2} d V-\frac{1}{V} \int_{S_{b}} u_{i} \tau_{\mathrm{bi}} d S_{b},
$$


is the dissipation including that as a result of bottom drag. Here, $S$ is the ocean surface area, $\rho_{0}$ is the reference density, $w$ is the vertical component of velocity, $K_{\nu}$ is the model eddy viscosity, $S_{b}$ is the surface area of the ocean bottom, and $\tau_{\text {bi }}$ is stress as a result of quadratic bottom drag.

Available potential energy $E_{a}$ is defined to be the difference between total gravitational potential energy $E_{p}$ and background potential energy $E_{b}$, calculated by adiabatically resorting the global stratification to be stably stratified and depending only upon height $z$. This procedure involves attributing a vertical position $z_{*}$ for each fluid parcel, which represents its location in the resorted profile, describing a hypothetical reference state [see Winters et al. (1995), for details], and leads to $E_{b}$ given by

$$
E_{b}=\frac{g}{\rho_{0} V} \int_{V} \rho z_{*} d V
$$

describing the minimum PE of the system. The APE (per unit mass) is then defined to be the difference between $E_{p}$ and $E_{b}$,

$$
E_{a}=\frac{g}{\rho_{0} V} \int_{V} \rho\left(z-z_{*}\right) d V .
$$

The APE tendency is

$$
\frac{d E_{a}}{d t}=\Phi_{z}+\Phi_{b}-\Phi_{d}
$$

where

$$
\Phi_{b} \equiv \frac{g}{\rho_{0} V} \int_{S}\left(z-z_{*}\right) Q d S
$$

is the power input by the surface buoyancy fluxes $Q$ and

$\Phi_{d}=\frac{g}{\rho_{0} V}\left[-\int_{V} K_{\rho} \frac{d z_{*}}{d \rho}\left(\frac{\partial \rho}{\partial x_{i}}\right)^{2} d V+\int_{V} K_{\rho} \frac{\partial \rho}{\partial z} d V\right]$,

the irreversible mixing and conversion of energy via diffusion of a stable gradient, respectively, where $K_{\rho}$ is the model eddy diffusivity. The surface buoyancy power input is composed of two terms, the first being the net input of gravitational potential energy by surface fluxes, $\Phi_{b_{1}}=\int_{S} z Q d S$ while the second component is the conversion of background to available potential energy by surface buoyancy fluxes, $\Phi_{b_{2}}=-\int_{S} z_{*} Q d S$. It should be noted that when the system is in an equilibrium state (i.e., when $\int_{S} Q d S=0$ in a time-averaged sense) then $\Phi_{b_{1}}=0$, meaning that $\Phi_{b}=\Phi_{b_{2}}$ (Hughes et al. 2009).
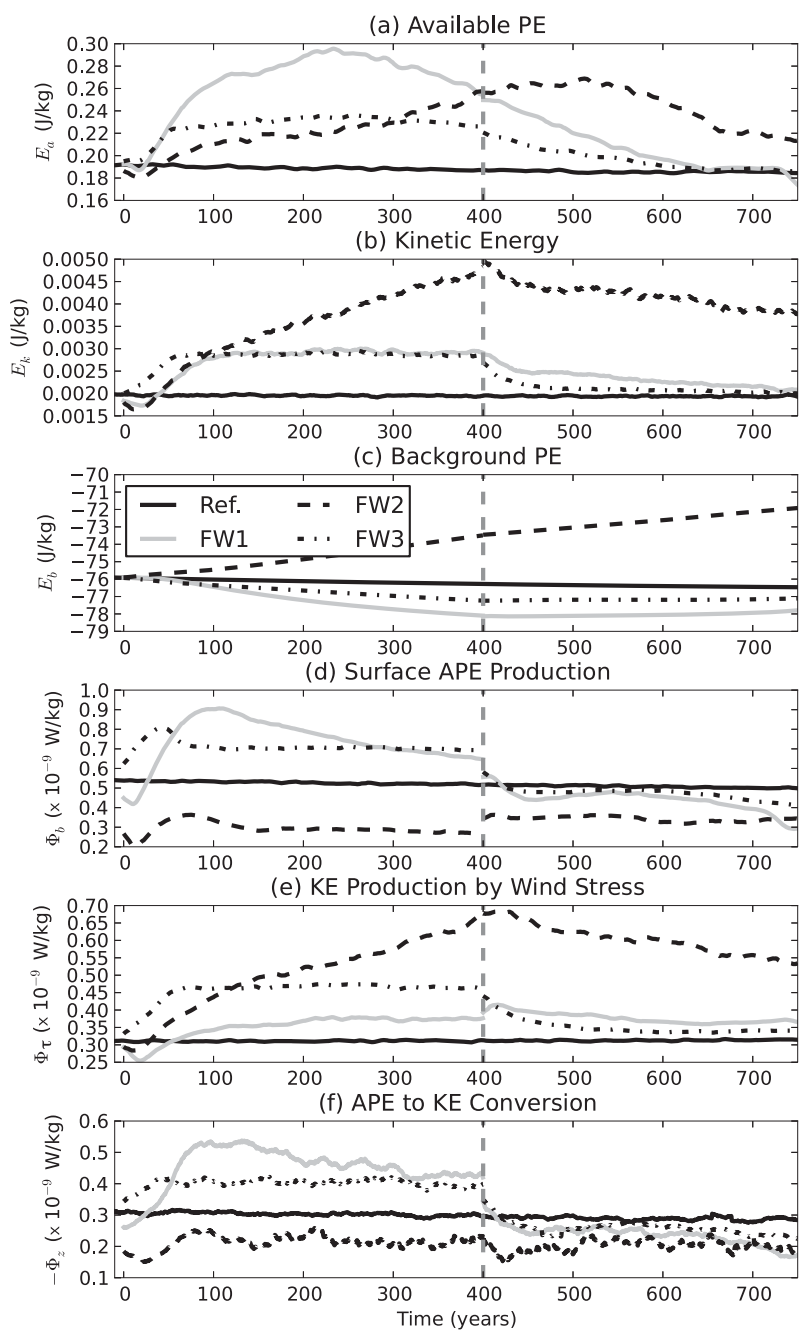

FIG. 5. Global energy diagnostics for the four simulations described here. (a) Available PE, (b) KE, (c) background PE, (d) surface buoyancy forcing of APE, (e) wind stress forcing of the KE, and (f) conversion from APE to KE by the resolved flow.

However, in transient simulations such as those shown here, the value of both buoyancy forcing components $\Phi_{b_{1}}$ and $\Phi_{b_{2}}$ depends on the arbitrary definition of the origin (as does $E_{p}$ ), while $E_{a}$ and $\Phi_{b}$ are independent of the reference frame. Specifically, if one elects to prescribe the origin $(z=0)$ at the ocean surface (as we do here) we again find that $\Phi_{b_{1}}=0$, irrespective of net surface buoyancy forcing. Thus, failing to break up potential energy into available and background components completely obscures the energetic role of surface buoyancy forcing.

Figure 5 shows time series of energy, as well as power input and conversion from the four simulations conducted here. In the reference case (solid line), $E_{a}$ (Fig. $5 a)$ is an order of magnitude larger than $E_{k}$ (Fig. 5b), and 
both are steady showing that the system is in equilibrium. Here, $E_{b}$ (Fig. 5c) is larger again, but (like $E_{p}$ ) is an arbitrary quantity, as it depends on the definition of the origin and is negative in these simulations; $E_{b}$ is also further from equilibrium because of the slow spinup of the deep ocean stratification. Sources of energy include power input from wind stress to $E_{k}$ (Fig. 5e) and surface buoyancy forcing input to $E_{a}$ (Fig. 5d) - these terms are the same order of magnitude (with APE input being just under a factor of 2 larger). About $50 \%$ of the APE input from surface buoyancy forcing is converted from APE to kinetic energy in the equilibrium state (Fig. 5f). Thus, the kinetic energy input is equally partitioned between wind stress input and conversion from APE, consistent with the results of Saenz et al. (2012), illustrating the importance of buoyancy forcing in the mechanical energy budget.

All three freshwater perturbations applied here (Fig. 5) generate an increase in both APE and kinetic energy (with a small decrease in the first decade of the perturbation) that persists for at least 400 years after forcing is adjusted back to the reference configuration. In the FW1 case, there is also an increase in surface APE production (i.e., $\Phi_{b}$ ), wind power input (i.e., $\Phi_{\tau}$ ), and the rate of conversion from APE to kinetic energy (i.e., $\Phi_{z}$ ). These increases in energy storage and production occur despite the marked reduction in the northern overturning streamfunction. The results imply that the global energy balance is a poor indicator of the strength of the Atlantic MOC. The total kinetic energy is most closely related to circumpolar transport. Surface APE input, and hence from APE to kinetic energy conversion are also likely to be dominated by Southern Ocean processes. Thus, we infer that a global analysis provides little insight into the energetics of a collapsing MOC, motivating the application of a regional energy analysis outlined in the following subsection.

The FW2 case, where freshwater fluxes are not compensated elsewhere in the domain, shows several key differences with respect to case FW1. First, surface APE production and consequent conversion to kinetic energy both reduce markedly, implying that reductions in the northern APE production are no longer offset by APE generation in the south. Second, kinetic energy increases rapidly in concert with circumpolar transport (because of a stronger thermal wind across the circumpolar channel) resulting in enhanced wind power input. Finally, despite the reduced APE power input the APE reservoir increases owing to subtle changes in the balance of terms controlling APE: the dissipation of APE by mixing and diffusion (not shown) is reduced and conversion of wind energy input to APE (which acts to weaken the APE to KE conversion) occurs.
The FW3 case, in which the freshwater perturbation is applied to the Southern Ocean, shows broadly similar energetic responses to FW1. This occurs despite the distinct dynamical response, most notably the strengthening of the northern overturning cell.

The interpretation of global energetics in this case is obviously complicated by the fact that major currents respond differently to forcing changes. The result is that the global energy balance may be dominated by one region or current, and thus global energy input does not constrain the behavior of the overturning circulation. An alternative approach is to examine the local energy density. This exercise would be trivial for kinetic energy, but finding the APE density is complicated by the fact that the integrand in (7) is not positive definite. However, several recent papers have outlined ways in which APE density may be calculated, and we proceed to apply these new techniques to the current simulations in the following section.

\section{b. APE density}

Recent attempts to outline the utility of APE density (Holliday and McIntyre 1981) include Roullet and Klein (2009), Molemaker and McWilliams (2010), and Winters and Barkan (2012). Here, we follow the notation of Winters and Barkan (2012), but note that each of these three implementations are physically equivalent. We define APE density $\mathcal{E}_{a}$ to be

$$
\mathcal{E}_{a}(x, y, z, t) \equiv\left(z-z_{*}\right)\left[\rho-\bar{\rho}\left(z, z_{*}\right)\right] \geq 0,
$$

where

$$
\bar{\rho}\left(z, z_{*}\right)=\frac{1}{\left(z-z_{*}\right)} \int_{z_{*}}^{z} \rho\left(z_{*}^{\prime}\right) d z_{*}^{\prime}
$$

The key point of this definition is that it allows us to write

$$
E_{a}=\frac{g}{\rho_{0} V} \int_{V} \mathcal{E}_{a} d V
$$

and thereby to analyze the regional variations of APE through a positive definite term.

We plot the zonally integrated APE density (scaled by $\left.g / \rho_{0}\right)$ for the reference case, averaged over the last 100 years of the simulation in Fig. 6a. Only the upper $1500 \mathrm{~m}$ of the ocean is shown, as APE density variations below this level are minimal on this time scale. Note that both dense water close to the surface (especially in the Southern Ocean) and lighter tropical water at the base of the thermocline (the latter of which sits higher in the stratification after resorting) records a positive APE 
(a) Reference

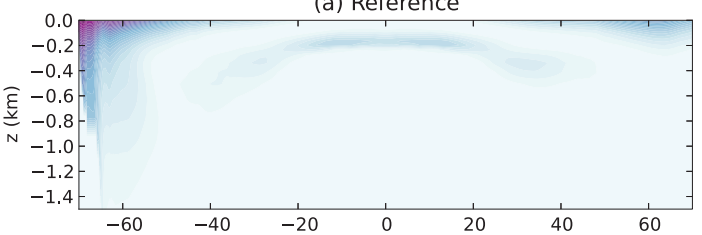

(b) FW1 - Ref.

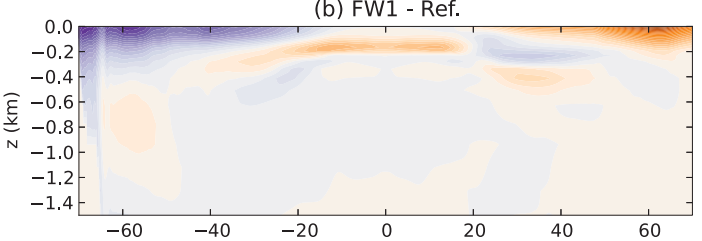

(c) FW2 - Ref.

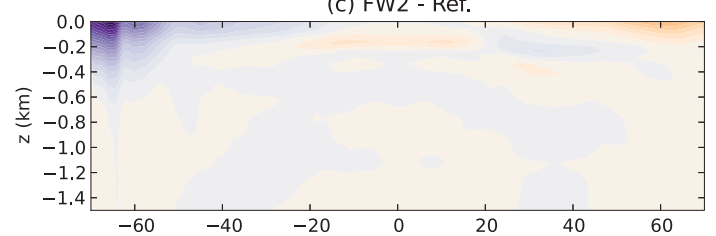

(d) FW3 - Ref

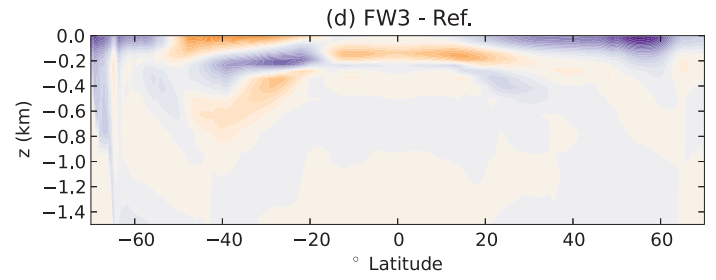

FIG. 6. (a) Zonally integrated and time-averaged APE density in the upper $1500 \mathrm{~m}$ for the last 100 years of the reference case [normalized by $g / \rho_{0}$ to give units in $10^{8} \mathrm{~J}(\mathrm{~m} \mathrm{~kg})^{-1}$ ]. Subsequent panels show the difference in APE density between the perturbed cases and the reference case for (b) FW1, (c) FW2, and (d) FW3. Note the different color scale for each panel.

density. The APE is concentrated in the Southern Ocean regions, with another maximum near the northern boundary.

When the compensating northern freshwater perturbation (FW1) is applied the difference in APE density shows a strong hemispheric asymmetry (Fig. 6b). The freshwater perturbation entirely eliminates the local maximum of APE density in the northern part of the basin (hence the absence of deep sinking in this simulation). However, the compensating salinity anomalies in the southern part of the basin result in enhanced APE density in the dominant Southern Ocean region. The net effect by $t=400$ years is an increase in total APE of about $30 \%$ (Fig. 5a), an increase in ACC transport and kinetic energy on the order of $50 \%$ (Figs. $3 \mathrm{a}$ and $5 \mathrm{~b}$ ) but a cessation of northern overturning (Figs. $3 b$ and $4 b$ ). It should be noted that the circulation does not reach a new equilibrium state after 400 years, and flow is still adjusting.

The APE density change from the last 100 years of the noncompensating northern freshwater perturbation
(FW2) is shown in Fig. 6c. Note that the scale here is triple the size of that for the FW1 case, despite a substantial decrease in the net power input from surface buoyancy forcing (Fig. 5d). This case differs primarily because of the net input of freshwater to the system, which acts to inhibit the sinking of APE through mixing, as well as enhanced wind power input reducing the APE to kinetic energy conversion term.

The third perturbation case (FW3) where freshwater forcing is applied at the latitudes of the circumpolar current is shown in Fig. 6d. This perturbation acts to simultaneously reduce APE density in the surface of the southern midlatitudes while increasing APE density in the polar latitudes of both hemispheres. The result is a substantial increase in all power sources (Fig. 5) and amplification of both overturning cells.

The distribution of APE throughout the domain makes it clear that the first-order effect of freshwater forcing is to reduce the near-surface APE density in the region of freshwater forcing. This effect can be quantified by examining the latitudinal variations of the surface buoyancy flux term, denoted $\phi_{b}$ below. That is, we define

$\phi_{b}(y)=\frac{g}{\rho_{0} V} \int\left(z-z_{*}\right)\left(Q_{T}+Q_{S}\right) d x=\phi_{b_{T}}+\phi_{b_{S}}$,

where $Q_{T}$ is the surface buoyancy forcing from heat fluxes and $\phi_{b_{T}}(y)$ the power input to APE from this process; $Q_{S}$ is the surface buoyancy forcing from freshwater terms with power input $\phi_{b_{S}}(y)$; and $Q_{T}+Q_{S}=Q$. This framework allows us to separate both the latitudinal and heat/freshwater contributions to the APE budget, as shown in Fig. 7. As expected, the total surface buoyancy forcing input to APE (Fig. 7c) is negligible in the northern part of the basin in the two northern freshwater flooding cases (FW1 and FW2), while both compensating cases (FW1 and FW3) have amplified APE input south of the circumpolar current. There is a clear correlation between local APE generation in each case, APE density near the surface and the strength of the corresponding overturning circulation cell, supporting the notion that APE generation is an important energetic control on the meridional overturning circulation.

Figure 7 also shows that the rate generation of APE by freshwater buoyancy fluxes $\phi_{b_{S}}$ is a small fraction of the total rate of generation of APE $\phi_{b}$ and is generally negative (because of freshening in polar regions). However, freshwater fluxes affect the dynamics of the circulation and the rate of generation of APE. Even in the northern part of the domain, where large freshwater fluxes are applied in cases FW1 and FW2, the primary 
(a) Temperature

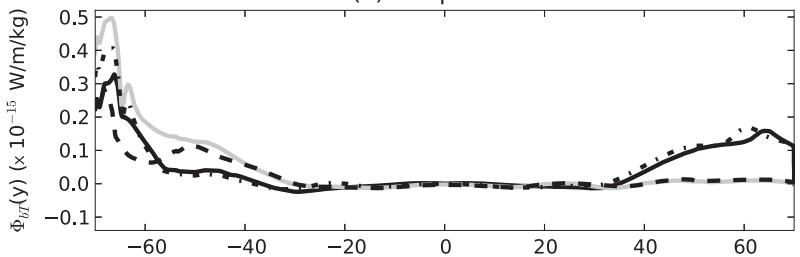

(b) Freshwater

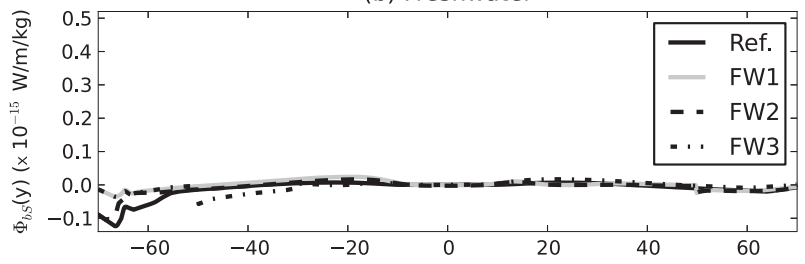

(c) Total

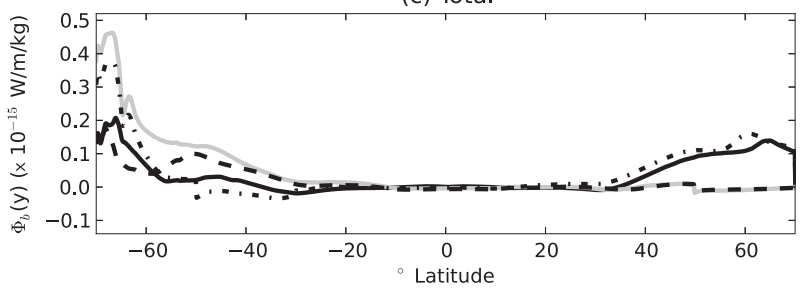

FIG. 7. Zonally integrated surface buoyancy forcing power input to APE as a function of latitude, averaged over the last 100 years of each case. (a) Contribution from heat fluxes, (b) contribution from freshwater fluxes, and (c) total APE generation (heat + freshwater).

reduction in local APE power input (when compared with the reference case) comes not from greater extraction of APE by freshwater contributions (Fig. 7b), but from the decreased heat flux power input (Fig. 7a). This occurs because the local APE production in (14) depends upon the product of local buoyancy anomaly (as measured by $z-z_{*}$ ) with buoyancy flux. Freshwater fluxes act on both of these terms, directly increasing the flux of buoyancy, but also progressively reducing $z-z_{*}$ as buoyancy increases. Figure 7 shows that the primary difference in northern APE generation between the control and FW1/FW2 cases is due to the reduction in $z-z_{*}$, which acts to decrease APE production by thermal processes (Fig. 7a).

The relative roles of heat and freshwater contributions are clarified by averaging surface buoyancy forcing input over latitudes greater than $40^{\circ} \mathrm{N}$ and plotting the transient response to the perturbations over the first 80 years of the simulation in Fig. 8. When the perturbation is switched on, the northern flooding cases (FW1 and FW2) show an instantaneous decrease of the total APE input to one-third of the reference case (Fig. 8c); this reduction is achieved entirely through the flux of buoyancy into the ocean by the imposed freshwater flux (Fig. 8b). From this point forward, power input resulting

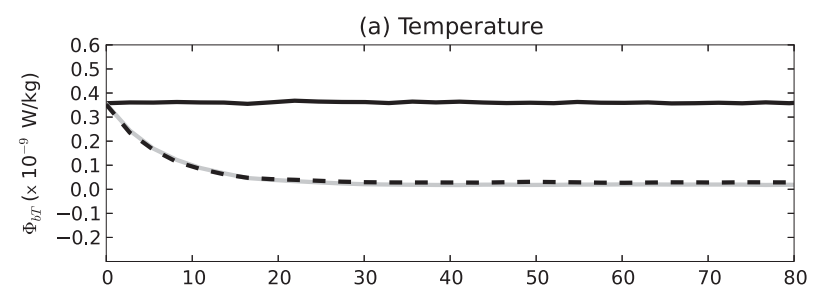

(b) Freshwater

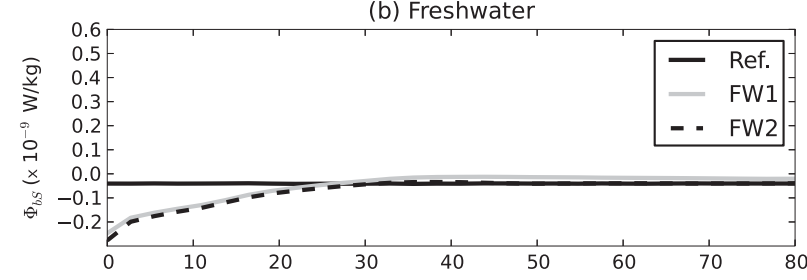

(c) Total

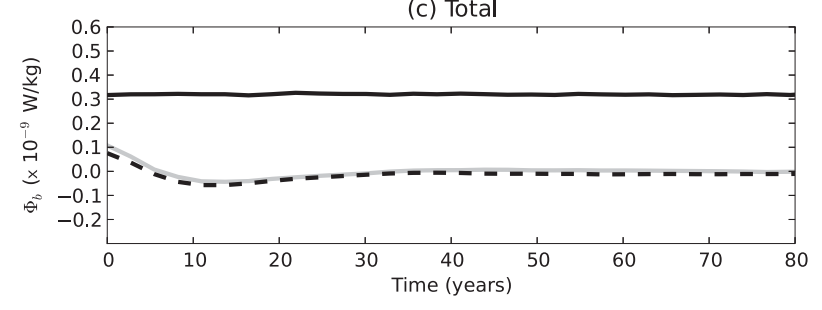

FIG. 8. Surface buoyancy forcing of APE, integrated over the region north of $40^{\circ} \mathrm{N}$ for the first 80 years of the reference and northern perturbed cases (FW1 and FW2). (a) Contribution from heat fluxes, (b) contribution from freshwater fluxes, and (c) total APE generation (heat + freshwater).

from both heat and freshwater approximately balance each other, with both terms decaying toward zero after 20 years (Figs. 8a,b). Given that the freshwater part of the buoyancy flux (i.e., $Q_{S}$ ) is entirely constant over this interval it is clear that the reduction in power input from surface cooling is dominated by a reduction in the $\left(z-z_{*}\right)$ term, rather than a decrease in the heat flux (which decreases by approximately a factor of 2; not shown). Thus, the process leading to the shutdown of the overturning circulation in this model is, initially an extraction of APE due to freshening, followed by a change in local density which minimizes the local value of $z-z_{*}$. Because $z \approx z_{*}$, buoyancy extraction (either thermal or haline) cannot substantially contribute the generation of APE in this region [see (14)].

\section{Discussion and conclusions}

There has been considerable debate in recent years regarding the mechanisms that control the meridional overturning circulation in the oceans. There appears to be no clear consensus in the field as to whether the overturning circulation should be considered to be driven by: (i) buoyancy from dense water formation in the polar regions; (ii) wind and tidal forcing; or (iii) both. 
If one defines the mechanical energy to be the combination of APE and kinetic energy, then from applying these ideas to equilibrium simulations of the overturning, it seems clear that both surface buoyancy fluxes and interior mixing (enhanced by winds and tides through internal gravity waves) play important roles in controlling the mechanical energy budget of the overturning circulation.

The primary question of interest addressed in the present paper is how the ocean responds to changes in surface buoyancy forcing. We use freshwater flux perturbations of the northern polar ocean to attempt to shut down the upper cell of the MOC, and analyze the results in an energetics framework. Our simulations are highly idealized, in the sense that we have a simple rectangular domain with minimal topography that spans only $40^{\circ}$ in longitude. We employ idealized forcing (relaxation to SST and prescribed freshwater fluxes) with no annual cycle or variability. However, the model has the advantage of being eddy permitting, running without explicit eddy parameterizations, being relatively well equilibrated and possessing the primary features of the overturning circulation: a (somewhat shallow) upper cell representing North Atlantic Deep Water formation, a fraction of which upwells in the Southern Ocean, and an abyssal cell representing Antarctic Bottom Water circulation.

Freshwater forcing introduced in the northern part of the domain efficiently reduces the strength of the upper cell of the meridional overturning circulation, as it does in a raft of previous simulations (e.g., Stouffer et al. 2006). For the parameter range investigated here, we do not appear to be in the multiple equilibria regime (Stommel 1961), but the model does possess a considerable transient asymmetry, with a rapid shutdown of the MOC (within decades) followed by a slow, multicentennial return once the original forcing is restored. We also test the concept of a freshwater perturbation in the southern part of the ocean basin, which acts to enhance the upper cell and particularly upwelling in the Southern Ocean.

The global energy balance of these transient simulations turns out to be particularly unilluminating. While the meridional overturning weakens in both northern freshwater hosing cases, most global energy metrics are enhanced. The APE power input is greater in the freshwater compensating (FW1) case because of stronger Southern Ocean contributions. The circumpolar transport increases in proportion to the thermal wind induced by a meridional salinity gradient, resulting in higher wind power input and an increase in kinetic energy, consistent with Saenz et al. (2012). The result is that almost all terms in the global mechanical energy budget are increased, while the upper overturning cell is shut down, implying that global energetics are a poor indicator of MOC strength.

A better understanding of the system can be obtained using APE density (Holliday and McIntyre 1981; Roullet and Klein 2009; Molemaker and McWilliams 2010; Winters and Barkan 2012). This is one of the first applications of this technique to ocean models, and it is particularly revealing. The APE density analysis shows that freshwater input acts to reduce the local rate of generation of APE. Moreover, it appears that surface APE density is a good indicator of the strength of the overturning cell associated with a given region (in both northern and southern hemispheres), reinforcing the notion of surface buoyancy forcing in providing energy for the overturning circulation. These findings are consistent with those of Saenko (2013) who uses the Lorenz approximation for APE to calculate the ocean's energetics response to increased $\mathrm{CO}_{2}$ in a global climate model.

The behavior of the idealized model used here also clarifies the role of local APE production in governing the temporal asymmetry of the response to surface buoyancy perturbations. Local APE production is the product of the buoyancy forcing (i.e., $Q$ ) and the local buoyancy anomaly (as measured by $z-z_{*}$ ). Initially, the freshwater perturbation acts directly to reduce $Q$, but also progressively reduces the difference between $z$ and $z_{*}$. For a large perturbation in $Q$ the system may reach a state where $z \approx z_{*}$, in which local APE input from either thermal or freshwater forcing is negligible. When the original forcing is reestablished, the system takes centuries (on the overturning time scale) to build up the density anomaly required to recreate the original APE production. Thus, while the shutdown of the MOC may be rapid, its reestablishment is necessarily slow.

The energetics of transient perturbations to the ocean's meridional overturning circulation is intrinsically linked with the local APE production by surface buoyancy forcing. As previously discussed, this result does not diminish the importance of the balance between mixing and dense water production in governing the overturning circulation, as proposed by Wunsch and Ferrari (2004). But it does clarify the mechanism by which future climate states, under which surface buoyancy forcing is likely to vary considerably, may generate abrupt responses in the meridional overturning circulation.

Acknowledgments. This paper stems from a visit by HAD to ANU, funded by the ARC Centre of Excellence for Climate System Science (CE11E0098). AMH and JAS were supported by Australian Research Council Grants DP0986244 and DP120102744. AMH was also supported by Australian Research Council Future Fellowship FT120100842. This research was undertaken on the NCI 
National Facility in Canberra, Australia, which is supported by the Australian Commonwealth Government.

\section{REFERENCES}

Borowski, D., R. Gerdes, and D. Olbers, 2002: Thermohaline and wind forcing of a circumpolar channel with blocked geostrophic contours. J. Phys. Oceanogr., 32, 2520-2540.

Clark, P. U., N. G. Pisias, T. F. Stocker, and A. J. Weaver, 2002: The role of the thermohaline circulation in abrupt climate change. Nature, 415, 863-869.

Gayen, B., R. W. Griffiths, G. O. Hughes, and J. A. Saenz, 2013: Energetics of horizontal convection. J. Fluid Mech., 716, R10, doi:10.1017/jfm.2012.592.

Hawkins, E., R. S. Smith, L. C. Allison, J. M. Gregory, T. J. Woollings, H. Pohlmann, and B. De Cuevas, 2011: Bistability of the Atlantic overturning circulation in a global climate model and links to ocean freshwater transport. Geophys. Res. Lett., 38, L10605, doi:10.1029/2011GL047208.

Hogg, A. M., 2010: An Antarctic Circumpolar Current driven by surface buoyancy forcing. Geophys. Res. Lett., 37, L23601, doi:10.1029/2010GL044777.

Holliday, D., and M. E. McIntyre, 1981: On potential energy density in an incompressible, stratified fluid. J. Fluid Mech., 107, 221-225, doi:10.1017/S0022112081001742.

Hughes, C. W., and C. Wilson, 2008: Wind work on the geostrophic ocean circulation: An observational study of the effect of small scales in the wind stress. J. Geophys. Res., 113, C02016, doi:10.1029/2007JC004371.

Hughes, G. O., and R. W. Griffiths, 2008: Horizontal convection. Annu. Rev. Fluid Mech., 40, 185-208, doi:10.1146/ annurev.fluid.40.111406.102148.

— A. M. Hogg, and R. W. Griffiths, 2009: Available potential energy and irreversible mixing in the meridional overturning circulation. J. Phys. Oceanogr., 39, 3130-3146.

Huisman, S. E., M. den Toom, H. A. Dijkstra, and S. Drijfhout, 2010: An indicator of the multiple equilibria regime of the Atlantic meridional overturning circulation. J. Phys. Oceanogr., 40, 551-567.

- H. A. Dijkstra, A. S. von der Heydt, and W. P. M. de Ruijter, 2012: Does net $E-P$ set a preference for North Atlantic sinking? J. Phys. Oceanogr., 42, 1781-1792.

Jones, D. C., T. Ito, and N. S. Lovenduski, 2011: The transient response of the Southern Ocean pycnocline to changing atmospheric winds. Geophys. Res. Lett., 38, L15604, doi:10.1029/ 2011 GL048145.

Kuhlbrodt, T., A. Griesel, M. Montoya, A. Levermann, M. Hofmann, and S. Rahmstorf, 2007: On the driving processes of the Atlantic meridional overturning circulation. Rev. Geophys., 45, RG2001, doi:10.1029/2004RG000166.

Marsh, R., D. Desbruyères, J. L. Bamber, B. A. de Cuevas, A. C. Coward, and Y. Aksenov, 2010: Short-term impacts of enhanced Greenland freshwater fluxes in an eddy-permitting ocean model. Ocean Sci., 6, 749-760, doi:10.5194/os-6-749-2010.

Marshall, J., A. Adcroft, C. Hill, L. Perelman, and C. Heisey, 1997: A finite-volume, incompressible Navier Stokes model for studies of the ocean on parallel computers. J. Geophys. Res., 102 (C3), 5753-5766.

Molemaker, M. J., and J. C. McWilliams, 2010: Local balance and cross-scale flux of available potential energy. J. Fluid Mech., 645, 295-314, doi:10.1017/S0022112009992643.
Morrison, A. K., A. M. Hogg, and M. L. Ward, 2011: Sensitivity of the Southern Ocean overturning circulation to surface buoyancy forcing. Geophys. Res. Lett., 38, L14602, doi:10.1029/ 2011 GL048031.

Munday, D. R., H. L. Johnson, and D. P. Marshall, 2013: Eddy saturation of equilibrated circumpolar currents. J. Phys Oceanogr., 43, 507-532.

Munk, W., and C. Wunsch, 1998: Abyssal recipes II: Energetics of tidal and wind mixing. Deep-Sea Res., 45, 1976-2009.

Paparella, F., and W. R. Young, 2002: Horizontal convection is non-turbulent. J. Fluid Mech., 466, 205-214.

Rahmstorf, S., 1995: Bifurcations of the Atlantic thermohaline circulation in response to changes in the hydrological cycle. Nature, 378, 145-149.

Roullet, G., and P. Klein, 2009: Available potential energy diagnosis in a direct numerical simulation of rotating stratified turbulence. J. Fluid Mech., 624, 45-55, doi:10.1017/S0022112008004473.

Saenko, O. A., 2013: Energetics of weakening and recovery of the Atlantic overturning in a climate change simulation. Geophys. Res. Lett., 40, 888-892, doi:10.1002/grl.50101.

Saenz, J. A., A. M. Hogg, G. O. Hughes, and R. W. Griffiths, 2012 Mechanical power input from buoyancy and wind to the circulation in an ocean model. Geophys. Res. Lett., 39, L13605, doi:10.1029/2012GL052035.

Scotti, A., and B. White, 2011: Is horizontal convection really "nonturbulent?" Geophys. Res. Lett., 38, L21609, doi:10.1029/ 2011 GL049701.

Shakespeare, C. J., and A. M. Hogg, 2012: An analytical model of the response of the meridional overturning circulation to changes in wind and buoyancy forcing. J. Phys. Oceanogr., 42, 1270-1287.

Smith, R. S., and J. M. Gregory, 2009: A study of the sensitivity of ocean overturning circulation and climate to freshwater input in different regions of the North Atlantic. Geophys. Res. Lett., 36, L15701, doi:10.1029/2009GL038607.

Stommel, H., 1961: Thermohaline convection with two stable regimes of flow. Tellus, 13, 224-230.

Stouffer, R. J., and Coauthors, 2006: Investigating the causes of the response of the thermohaline circulation to past and future climate changes. J. Climate, 19, 1365-1387.

Tailleux, R., 2009: On the energetics of stratified turbulent mixing, irreversible thermodynamics, Boussinesq models and the ocean heat engine controversy. J. Fluid Mech., 638, 339-382, doi:10.1017/S002211200999111X.

Toggweiler, J. R., and B. Samuels, 1998: On the ocean's largescale circulation near the limit of no vertical mixing. J. Phys. Oceanogr., 28, 1832-1852.

Weijer, W., M. E. Maltrud, M. W. Hecht, H. A. Dijkstra, and M. A. Kliphuis, 2012: Response of the Atlantic Ocean circulation to Greenland Ice Sheet melting in a strongly-eddying ocean model. Geophys. Res. Lett., 39, L09606, doi:10.1029/2012GL051611.

Winters, K. B., and R. Barkan, 2012: Available potential energy density for Boussinesq fluid flow. J. Fluid Mech., 714, 476-488.

_, P. N. Lombard, J. J. Riley, and E. A. D'Asaro, 1995: Available potential energy and mixing in density-stratified fluids. J. Fluid Mech., 289, 115-128.

Wolfe, C. L., and P. Cessi, 2011: The adiabatic pole-to-pole overturning circulation. J. Phys. Oceanogr., 41, 1795-1810.

Wunsch, C., and R. Ferrari, 2004: Vertical mixing, energy, and the general circulation of the oceans. Annu. Rev. Fluid Mech., 36, 281-314. 